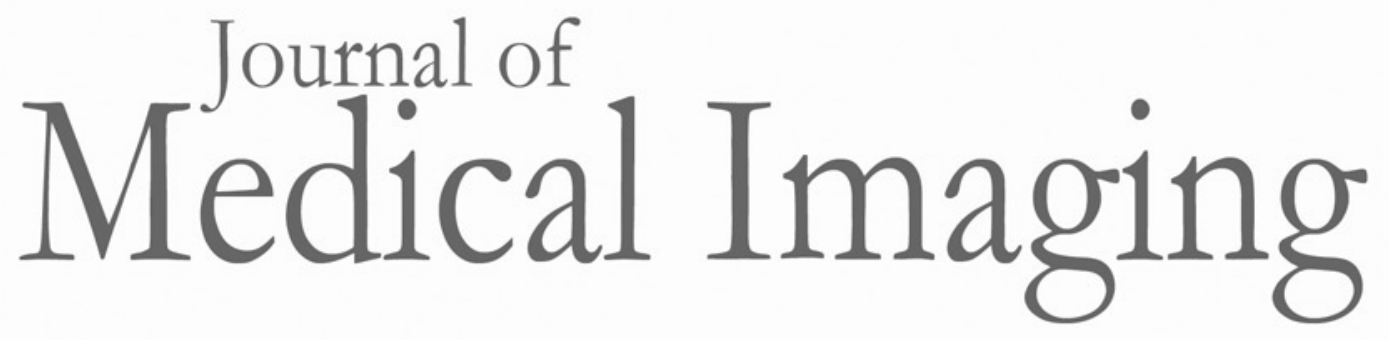

Medicallmaging.SPIEDigitalLibrary.org

\title{
QuantitativeT2: interactive quantitative T2 MRI witnessed in mouse glioblastoma
}

Tonima Sumya Ali

Thorarin Albert Bjarnason

Donna L. Senger

Jeff F. Dunn

Jeffery T. Joseph

Joseph Ross Mitchell

\section{SPIE.}




\title{
QuantitativeT2: interactive quantitative T2 MRI witnessed in mouse glioblastoma
}

\author{
Tonima Sumya Ali, ${ }^{a, \star}$ Thorarin Albert Bjarnason, ${ }^{b, c, d}$ Donna L. Senger, ${ }^{e}$ Jeff F. Dunn, ${ }^{f, g}$ \\ Jeffery T. Joseph, ${ }^{h}$ and Joseph Ross Mitchelli \\ ${ }^{a}$ Queensland University of Technology, Science and Engineering Faculty, Department of Biomedical Engineering and Medical Physics, \\ 2 George Street, Brisbane, QLD 4000, Australia \\ bDiagnostic Imaging Services, Interior Health, 101-3330 Richter Street, Kelowna V1W 4V5, Canada \\ CUniversity of British Columbia, Department of Radiology, 2329 W Mall, Vancouver V6T 1Z4, Canada \\ dUniversity of British Columbia Okanagan, 3333 University Way, Kelowna V1V 1V7, Canada \\ eUniversity of Calgary, Faculty of Medicine, Department of Oncology, 2500 University Drive, Calgary T2N 1N4, Canada \\ fUniversity of Calgary, Faculty of Medicine, Hotchkiss Brain Institute, 3330 Hospital Drive, Calgary T2N 4N1, Canada \\ IUniversity of Calgary, Faculty of Medicine, Department of Radiology, 2500 University Drive, Calgary T2N 1N4, Canada \\ ${ }^{\mathrm{h}}$ Foothills Medical Centre, Department of Pathology, 140329 Street, Calgary T2N 2T9, Canada \\ 'Mayo Clinic College of Medicine, Department of Radiology, 200 1st Street, Rochester, Minnesota 55905, United States
}

\begin{abstract}
The aim of this study was to establish an advanced analytical platform for complex in vivo pathologies. We have developed a software program, QuantitativeT2, for voxel-based real-time quantitative T2 magnetic resonance imaging. We analyzed murine brain tumors to confirm feasibility of our method for neurological conditions. Anesthetized mice (with invasive gliomas, and controls) were imaged on a 9.4 Tesla scanner using a Carr-Purcell-Meiboom-Gill sequence. The multiecho T2 decays from axial brain slices were analyzed using QuantitativeT2. T2 distribution histograms demonstrated substantial characteristic differences between normal and pathological brain tissues. Voxel-based quantitative maps of tissue water fraction (WF) and geometric mean T2 (gmT2) revealed the heterogeneous alterations to water compartmentalization caused by pathology. The numeric distribution of WF and gmT2 indicated the extent of tumor infiltration. Relative evaluations between in vivo scans and ex vivo histology indicated that the T2s between 30 and $150 \mathrm{~ms}$ were related to cellular density and the integrity of the extracellular matrix. Overall, QuantitativeT2 has demonstrated significant advancements in qT2 analysis with real-time operation. It is interactive with an intuitive workflow; can analyze data from many MR manufacturers; and is released as open-source code to encourage examination, improvement, and expansion of this method. ๑ The Authors. Published by SPIE under a Creative Commons Attribution 3.0 Unported License. Distribution or reproduction of this work in whole or in part requires full attribution of the original publication, including its DOI. [DOI: 10.1117/1.JMI.2.3.036002]
\end{abstract}

Keywords: magnetic resonance imaging; QuantitativeT2; ; qT2; software; glioblastoma.

Paper 15034R received Feb. 23, 2015; accepted for publication Jun. 9, 2015; published online Jul. 21, 2015.

\section{Introduction}

The soft tissue contrast in magnetic resonance imaging (MRI) results from the water-filled biological microcompartments. Few MRI relaxation parameters, such as spin-lattice (T1) and spinspin (T2) relaxation times, are designed to measure the distribution of water protons. $\mathrm{T} 2$ is specifically sensitive to the extent of water compartmentalization. ${ }^{1} \mathrm{~T} 2$ times decrease as tissue water loses magnetization to surrounding molecules. For example, white matter (WM) has a short T2 time, compared to gray matter (GM) and cerebrospinal fluid (CSF), since WM water is highly compartmentalized by myelin bilayers that promote magnetization exchange. Consequently, it is possible to identify distinct pools of water molecules in biological tissues according to their unique T2 times. When pathological conditions induce changes in tissue structures, significant deviations from normal T2s may occur.

A two-dimensional T2-weighted MR image, which is commonly used in clinical practice, is created by taking a snapshot of the T2 decay for each voxel of the imaging plane.

*Address all correspondence to: Tonima Sumya Ali, E-mail: tonima.ali@qut .edu.au
Quantitative T2 (qT2) imaging is more complex. It requires a Carr-Purcell-Meiboom-Gill (CPMG) imaging sequence ${ }^{2}$ that applies multiple refocusing pulses to generate a multiecho T2 image. With sufficient data points, pure T2-weighted decays are computed and further analyzed to determine the combination of $\mathrm{T} 2$ times that contributed to the measured decay. ${ }^{3}$ This allows scientists to create $\mathrm{T} 2$ distributions and discern microcompartmental structures. ${ }^{4}$ Multiexponential T2 decay analysis has been applied to study cartilage, ${ }^{5}$ blood,${ }^{6}$ muscle,${ }^{7}$ fat, ${ }^{7}$ cervix,${ }^{7}$ liver, ${ }^{8}$ breast, ${ }^{9}$ and prostate. ${ }^{10}$ In addition to medical applications, this method has been applied by food scientists to determine if cheese was made using raw or heat-treated milk, ${ }^{11}$ and as a quality control tool for fat distributions in fried food..$^{12}$ In the brain, qT2 has been used to identify normal $\mathrm{WM},{ }^{13} \mathrm{GM},{ }^{1,14}$ and tumor. ${ }^{15}$ This method has detected myelin content in multiple sclerosis ${ }^{16,17}$ and uncovered previously undetected water compartments in human brain pathology in vivo. ${ }^{18,19}$

The typical qT2 analysis proceeds as follows: (1) a multiecho T2 MR image is acquired; (2) a region of interest (ROI) is specified on the image; (3) decay data within the ROI are averaged; and (4) a T2 distribution is created by fitting a sum of weighted exponential decays to the averaged decay data. ${ }^{20}$ To make the fit more resilient to noise, a regularization routine is often applied. 
Although qT2 showed promise for characterizing pathological structures, the following limitations have discouraged widespread clinical adoption of qT2. First, the placement of the ROI requires prior knowledge of where pathology is located. Second, the average signal of an ROI may mask important physiological variability of complex biological tissues. Third, the regularization causes underestimation of the myelin water fraction (MWF), and this error increases as the signal-tonoise ratio decreases. ${ }^{20}$ Fourth, the analysis often requires multiple software programs with different interfaces and input requirements. The main objective of this study was to overcome these limitations and establish an advanced analytical platform for complex pathologies, in vivo.

For this purpose, we developed QuantitativeT2, an integrated tool for qT2 analysis. This new tool results from four novel contributions: (1) voxel-based multiexponential qT2; (2) voxelbased T2 distribution histograms; (3) interactive computation of quantitative parametric maps; and (4) numerical assessment of qT2 parameter maps for user-specified ROIs. We hypothesize that our method will improve the existing qT2 analysis. It should identify pathological tissues at early stages of disease with quantitative information on its progression. We analyzed an in vivo mouse model of human malignant glioma (MG) to assess its potentials. Diagnosis of MG is particularly challenging as it is diffuse, highly invasive, and patients rarely survive in the long-term. ${ }^{21}$ Animal MG models are commonly used to study the disease progression in a controlled environment. Although increased T2 times have been observed in mouse gliomas using conventional T2-weighted MRI, this method could not detect gliomas at early stages. ${ }^{22}$ Multiexponential T2 has been reported in rat glioblastoma, ${ }^{23}$ but the contributing $\mathrm{T} 2$ values or underlying factors for multiexponential behavior were not explored. Our method allowed probing at the subvoxel level to quantify pathological T2 with specific information on tumor infiltration. We generated quantitative parametric maps that identified water compartment alterations caused by glioma invasion and compared these results to histology.

\section{Materials and Methods}

\subsection{Animal Model}

The mouse MG model was established by implanting immunocompromised mice with patient-derived brain tumor initiating cells (BTICs), a subpopulation of brain tumor cells that maintain the ability to self-renew, proliferate, and give rise to differentiated daughter cells that repopulate the tumor. ${ }^{24}$ BTICs, when implanted into the brains of severe combined immunodeficiency (SCID) mice, result in highly invasive tumors that closely resemble MG in humans..$^{21,24,25}$ In this study, six-weekold female SCID mice (Charles River Laboratories, Ontario, Canada) were housed in groups of three and maintained on a 12-h light/dark schedule at a temperature of $22 \pm 1^{\circ} \mathrm{C}$ and a relative humidity of $50 \pm 5 \%$ for a period of 14 weeks. Food and water were available ad libitum. For the experimental group ( $n=3$, malignant glioma or MG group), the MG tumor model was established on day 3 by inoculating each mouse intracerebrally, in the right putamen with $1 \times 10^{4}$ BTICs collected from human surgical specimens. The control group ( $n=3$, control or C group) contained three, age-matched, normal SCID mice. All animal and human tissue protocols were reviewed and approved by University of Calgary Animal Care Committee.

\subsection{MRI Protocol}

All mouse brains ( $\mathrm{C}$ and MG groups) were scanned in vivo on day 93 following the BTIC inoculation. The MR imaging was carried out using a 9.4 Tesla (T) Bruker Avance system (Bruker, Billerica, Massachusetts). Four axial slices were imaged from each brain using a CPMG sequence. With a repetition time of $3000 \mathrm{~ms}$, 128 echoes were collected with $5.5 \mathrm{~ms}$ echo spacing and 4 averages. The slices were $0.75 \mathrm{~mm}$ thick, covering a $1.92 \mathrm{~cm}^{2}$ field of view. The image matrix contained $128 \times 128$ pixels. Each pixel represented signals from a voxel of $0.15 \times 0.15 \times 0.75 \mathrm{~mm}$ volume. MR data were saved in raw data format ( 8 bit unsigned). For each animal, the MR slice containing the largest cross-section of the mouse brain was selected for further analysis.

\subsection{T2 Decay Analysis}

Multiexponential decay analysis was carried out following welldescribed techniques ${ }^{26,27}$ applied most recently by our group ${ }^{20}$ for ROI-based analysis. Briefly, if one assumes that the T2 decay within a particular voxel is multiexponential, and can be decomposed into a summation of monoexponential functions, then the signal, $y$, measured at each of $N$ CPMG echoes can be modeled as

$y_{i}=\sum_{j=1}^{M} s_{j} \exp \left(-t_{i} / \mathrm{T} 2_{j}\right), \quad i=1,2, \ldots, N$

where $t_{i}$ are the CPMG echo times, $M$ is the number of T2 bins used to model the T2 decay (described in a subsequent paragraph), and $s_{j}$ are the relative weightings for each monoexponential function.

Contrary to the conventional practice, we solved this equation to determine individual $s_{j}$ weightings - once for each voxel in the MR slice. This intensive computation was executed in $\mathrm{C}$ language by representing Eq. (1) as an $N \times M$ matrix [with $N$ rows corresponding to the echo measurement times $\left(t_{i}\right)$ and $M$ columns corresponding to the T2 bins] as shown in Eq. (2). With preset $\mathrm{T} 2$ bins, the unknowns, $s_{j}$, were solved using iterative non-negative least-square (NNLS) algorithm by minimizing error between the model and the measured echoes.

$$
\begin{gathered}
{\left[\begin{array}{ccccc}
A_{1,1} & A_{1,2} & \ldots & A_{1, M-1} & A_{1, M} \\
A_{2,1} & & & & A_{2, M} \\
\ldots & \ldots & \ldots & \ldots & \ldots \\
A_{N-1,1} & & & & A_{N-1, M} \\
A_{N, 1} & A_{N, 2} & \ldots & A_{N, M-1} & A_{N, M}
\end{array}\right]\left[\begin{array}{c}
s_{1} \\
s_{2} \\
\ldots \\
s_{M-1} \\
s_{M}
\end{array}\right]} \\
=\left[\begin{array}{c}
y_{1} \\
y_{2} \\
\cdots \\
y_{N-1} \\
y_{N}
\end{array}\right] .
\end{gathered}
$$

In this study, the echo magnitude reached the noise floor at the 97 th echo. Therefore, $N$ was set to 96 and echoes 97 to 128 were not used to avoid analysis artifacts that can be caused by the Rician noise floor. ${ }^{28}$ In addition, $M=120$ logarithmically spaced T2 bins ranging from $8.25 \mathrm{~ms}(1.5 \times$ shortest echo 
time) to $1056 \mathrm{~ms}(2 \times$ longest echo time $)$ were used to model the $\mathrm{T} 2$ decay in each voxel. The $\mathrm{T} 2-s_{j}$ combinations for every voxel were stored independently as T2 distributions, and also summed together to create a T2 distribution histogram for the entire MR slice.

\subsection{Quantitative Parametric Mapping}

A T2 distribution is evaluated by gmT2 and WF measures. The gmT2 is mean T2 time on a log scale. ${ }^{29}$ The graphic user interface (GUI) of QuantitativeT2 is equipped with two slider bars that allowed selection of a $\mathrm{T} 2$ range in the $\mathrm{T} 2$ distribution histogram. For a range between $\mathrm{T} 2_{\text {low }}$ and $\mathrm{T} 2_{\text {high }}$, gmT 2 of each voxel for the cross-sectional image was calculated using the following formula:

$\mathrm{gmT} 2=\exp \left(\sum_{\mathrm{T} 2_{\text {low }}}^{\mathrm{T} 2_{\text {high }}} s_{j} \log \left(\mathrm{T} 2_{j}\right) / \sum_{\mathrm{T} 2_{\text {low }}}^{T 2_{\text {high }}} s_{j}\right)$.

The gmT2 values were arranged in a parametric map, superimposed on a gray-scale T2 scan and displayed on upper left panel (Figs. 1 and 2). WF value indicates the relative signal strength of a T2 range with respect to the entire T2 distribution. ${ }^{1}$ QuantitativeT2 computed WF values individually for each voxel using the following formula:

$\mathrm{WF}=\sum_{\mathrm{T} 2_{\text {low }}}^{\mathrm{T} 2_{\text {high }}} \operatorname{area}\left(s_{j}\right) / \sum_{\mathrm{T} 2_{\text {min }}}^{\mathrm{T} 2_{\text {max }}} \operatorname{area}\left(s_{j}\right)$,

where $\mathrm{T} 2{ }_{\text {low }}$ and $\mathrm{T} 22_{\text {high }}$ defined the selected $\mathrm{T} 2$ range, and $\mathrm{T} 2_{\text {min }}$ and $\mathrm{T} 22_{\max }$ were the minimum and maximum $\mathrm{T} 2$ times in the $\mathrm{T} 2$ distribution histogram. Area $\left(s_{j}\right)$ referred to the T2 distribution histogram area, made up of discrete $s_{j}$ weightings, between the defined T2 times. The WF map was then superimposed on a gray-scale T2 scan and displayed in upper right panel (Figs. 1 and 2). The gray-scale scans are used for anatomical reference. We implemented flexible color mapping and window/leveling algorithms for improved visualization. A sketch function was implemented for both parametric maps for the user to draw an arbitrary ROI for further analysis. A local T2 distribution histogram was computed from the $\mathrm{T} 2$ data only within the user-defined ROI. These distributions were scaled for display such that the area under the distribution curve was 1 and represented $100 \%$ of the signals.

\subsection{Histological Analysis}

The animals were immediately sacrificed following MRI. The brain from each animal was collected, fixed with $4 \%$ paraformaldehyde, paraffin-embedded, and cut into 5- $\mu \mathrm{m}$-thick slices. For each mouse, a whole brain tissue section corresponding to the MR scan segment was stained for nestin, using an antibody against this human neural cell progenitor protein $^{25}$ (SigmaAldrich, Oakville, Ontario, Canada). Hematoxylin was used as a nuclear counter stain. A second tissue section within the MR scan slice was stained with hematoxylin and eosin (H\&E) (EMD Chemicals, Gibbstown, USA; Sigma-Aldrich, Oakville, Ontario, Canada).

The quantitative analysis of the nestin stained histology sections was performed using the ACIS III automated cellular imaging system. It consisted of five steps: (1) the histology slide was digitized using ACIS III software; (2) the digital section was compared against the segmented gmT2 map of the same mouse, and histological regions corresponding to the $\mathrm{T} 2$ bands were identified; (3) within the histological region for each T2 band, four circular ROIs, each with a diameter of $500 \mu \mathrm{m}$, were specified using ACIS III; (4) the percentage fraction of brown area (nestin stained, tumor cells) was calculated for each ROI; and (5) the average percentage was computed for the four ROIs

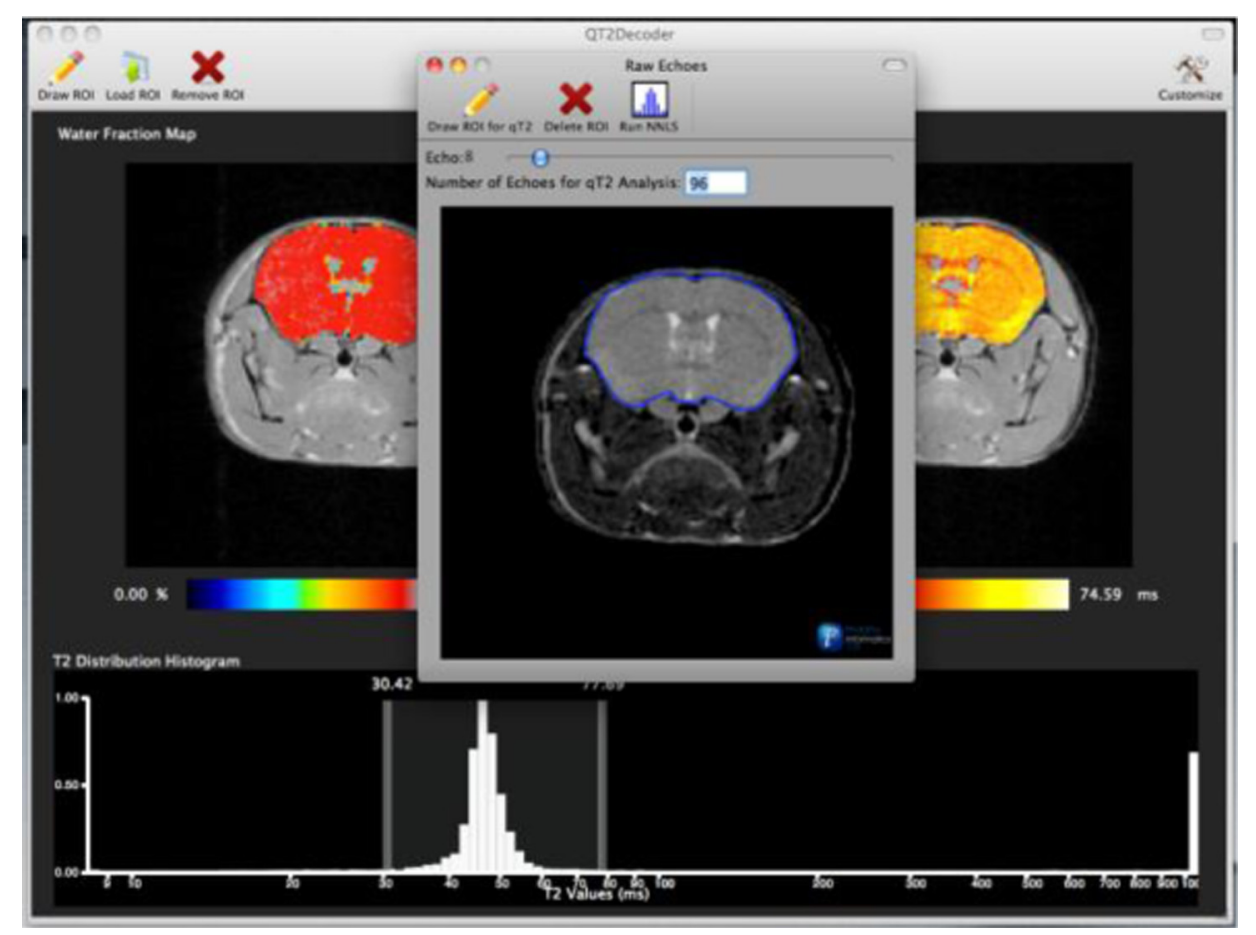

Fig. 1 Analysis workflow of QuantitativeT2 for a control mouse brain C1. (Video 1) (MOV, 1.86 MB) [URI: http://dx.doi.org/10.1117/1.JMI.2.3.036002.1]. 




Fig. 2 Analysis workflow of QuantitativeT2 for a glioma induced mouse brain MG1. (Video 2) (MOV, 1.25 MB) [URI: http://dx.doi.org/10.1117/1.JMI.2.3.036002.2].

corresponding to each T2 band. Data were collected from three control mice and three experimental mice by repeating the same process.

\subsection{QuantitativeT2 Architecture and Workflow}

QuantitativeT2 was developed and tested on an Apple Mac Pro running Mac OS X 10.6. The Mac Pro was equipped with $3 \mathrm{~GB}$ of $1066 \mathrm{MHz}$ DDR3 ECC SDRAM and powered by two $2.93 \mathrm{GHz}$ Quad Core 45-nm Xeon W3540 processors. QuantitativeT2 used the C programming language for the computationally intensive voxel-based qT2 and the Objective C programming language for data management. The GUI was developed using Objective $\mathrm{C}$ and Interface Builder. The software components were organized according to the model view controller (MVC) architectural pattern and were compiled by Xcode (version 3.2). MVC isolated the application logic from data input and presentation. This allowed independent development and maintenance of the data processing and user interface units. Our matrix-fitting algorithm was written in the $\mathrm{C}$ programming language; it incorporated the open-source NNLS code developed and validated by Deguet. ${ }^{30}$

Analysis in QuantitativeT2 followed a simple three-step approach. Step 1: the system performed voxel-based qT2 decay analysis for all voxels within the brain region. This produced a T2 distribution histogram, or a signature, that illustrated the general T2 properties of the tissue cross-section. The pathological T2 values may be visible in the distribution. Step 2: the user selected a T2 range from the histogram using slider bars. The system computed and displayed gmT2 and WF maps for the selected range in real time. These maps helped localize regions with pathological T2. Step 3: the user specified an ROI guided by the gmT2 map values and the extent of heterogeneity on the WF map. The mean and standard deviation of WF and gmT2 data within the ROI were then computed and saved.

\section{Results}

QuantitativeT2 has introduced a comprehensive voxel qT2 analysis method, by combining novel and existing features, which is intuitive and effective for disease diagnosis. Figure 3 shows proton density (PD) weighted and T2-weighted scans of $\mathrm{C} 1$ and MG1 as obtained from MR scanner. These scans are commonly used in clinical practice. Although informative, it is difficult to interpret these scans and isolate pathological tissues. Figures 1 and 2 show the analysis by QuantitativeT2 from raw MR data to parametric maps and numerical results for $\mathrm{C} 1$ and MG1.

\subsection{T2 Distribution Histograms of $C$ and MG Groups}

Figure 4(a) displays the T2 distribution histogram computed from $\mathrm{C} 1$. Most of the signal was concentrated in a single peak centered at $47.6 \mathrm{~ms}$ with tightly packed values in the 30.4 to $60.8 \mathrm{~ms}$ range. A second peak corresponding to CSF was observed at $1056 \mathrm{~ms}$. Figure 5(a) displays the T2 distribution histogram computed for MG1. The histogram was multimodal and shifted toward longer T2 compared to the histogram of C1. Three T2 peaks were observed centered at 51.7, 60.8, and $84.3 \mathrm{~ms}$ suggesting the presence of at least three different tissue types. A CSF peak was also observed at 1056 ms. For a comparison with control $\mathrm{C} 1$, the $\mathrm{T} 2$ values in this distribution were divided into two ranges: low (30.4 to $60.8 \mathrm{~ms}$, same as $\mathrm{C} 1$ ) and high (63.4 to $149.2 \mathrm{~ms}$, higher than $\mathrm{C} 1)$.

\subsection{Tumor Segmentation by T2-Specific Mapping}

Figures 4(b) and 4(c) display the gmT2 and the WF maps computed for the low T2s for mouse $\mathrm{C} 1$. The gmT2 map selectively displayed tissues corresponding to the $\mathrm{T} 2$ range specified in Fig. 4(a). It displayed the average $\mathrm{T} 2$ value, within the $\mathrm{T} 2$ 
(a)

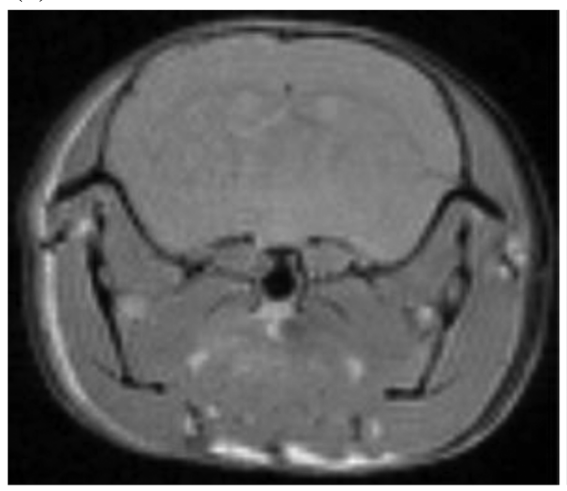

(c)

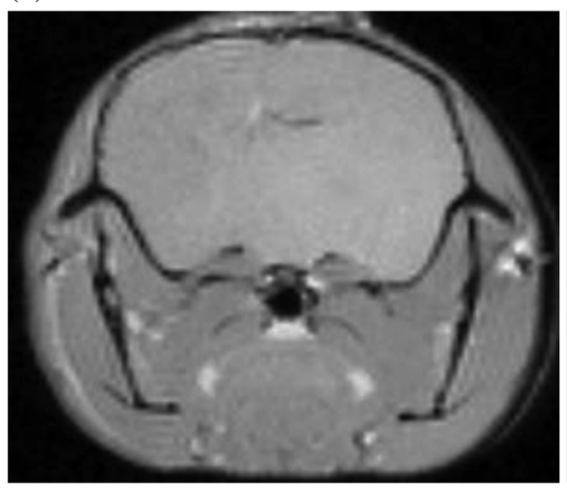

(b)

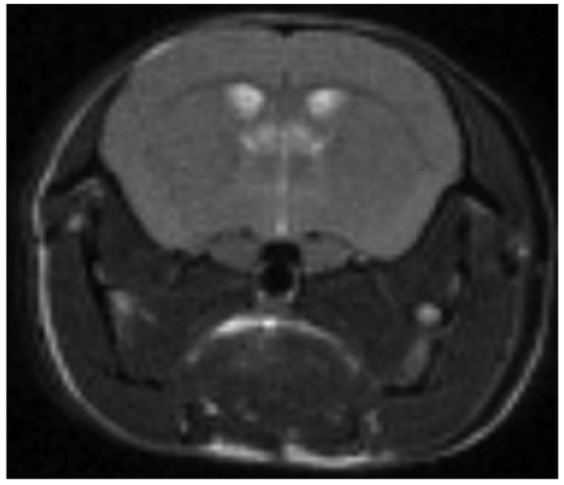

(d)

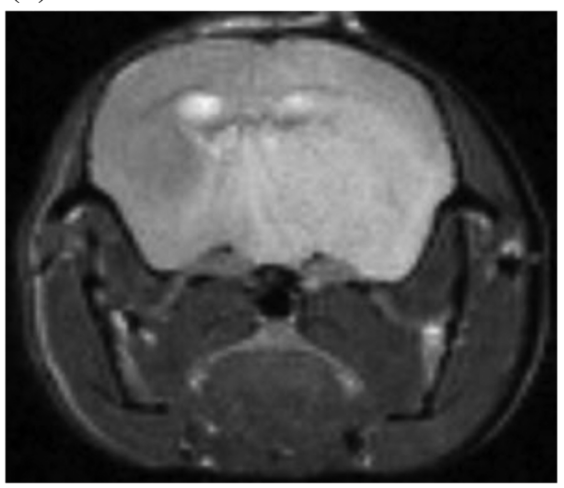

Fig. 3 (a) Proton density weighted and (b) T2-weighted axial brain slice of control C1. (c) Proton density weighted and (d) T2-weighted axial brain slice of glioma induced MG1.

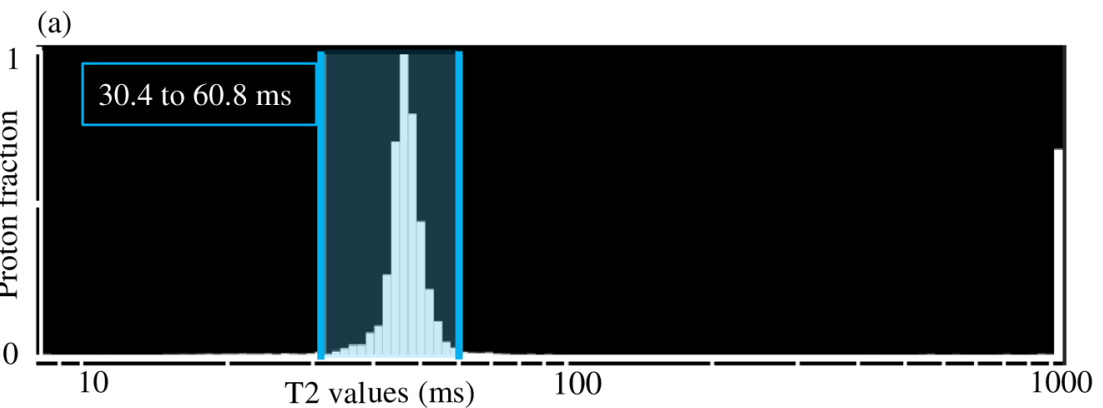

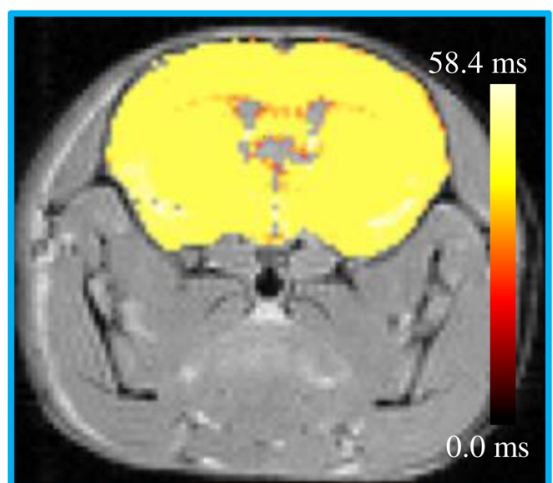

(b)

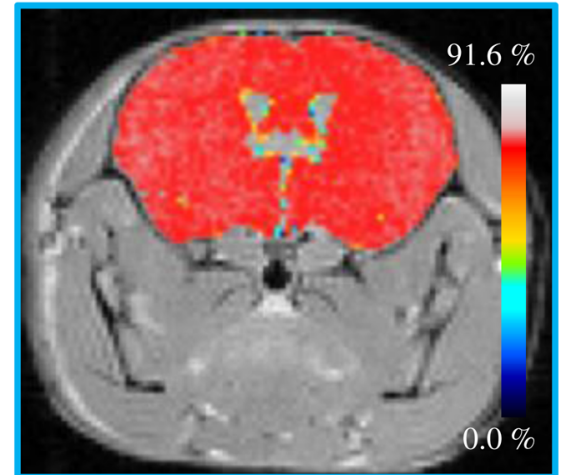

(c)

Fig. 4 Quantitative T2 (qT2) analysis results of control C1 by QuantitativeT2. (a) The T2 distribution histogram for a single axial slice of $\mathrm{C} 1$, (b) the geometric mean T2 (gmT2) map, and (c) water fraction (WF) map computed for the T2 range 30.4 to $60.8 \mathrm{~ms}$ as specified in the histogram. 
(a)

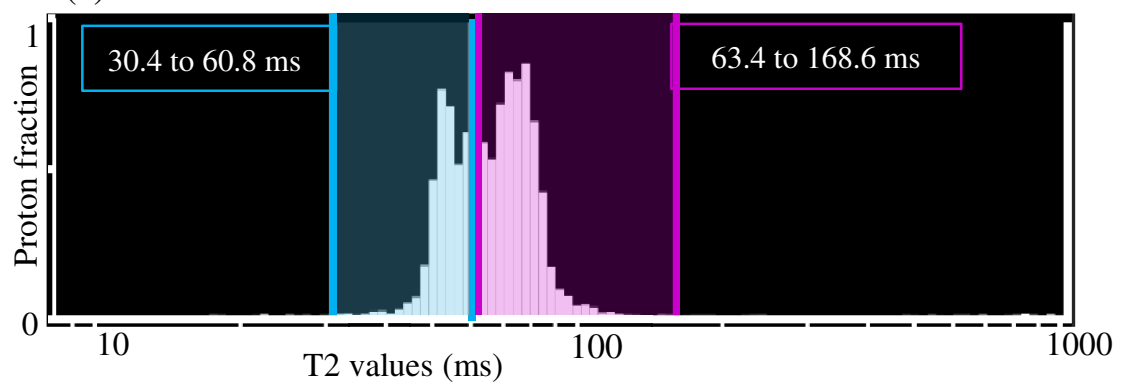

(b)

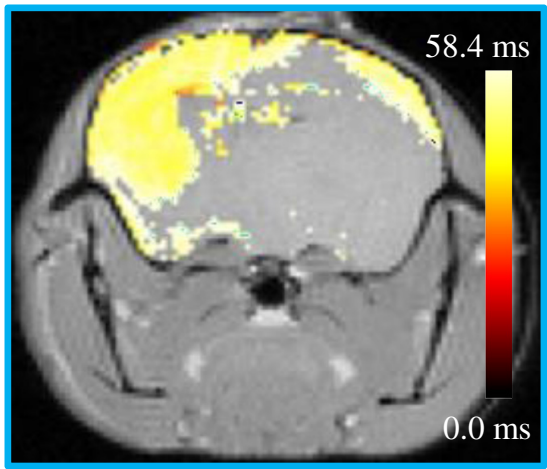

(d)

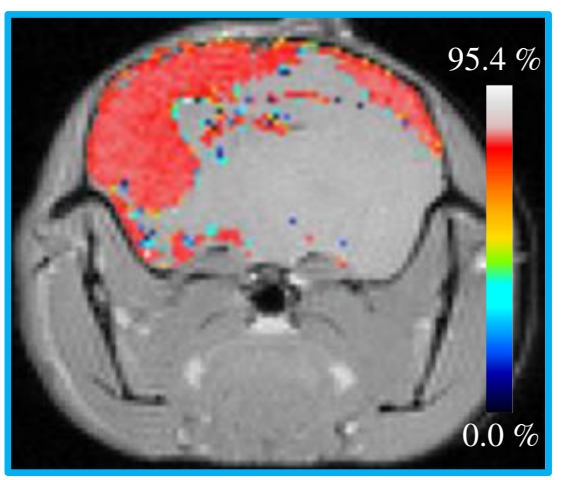

(f)

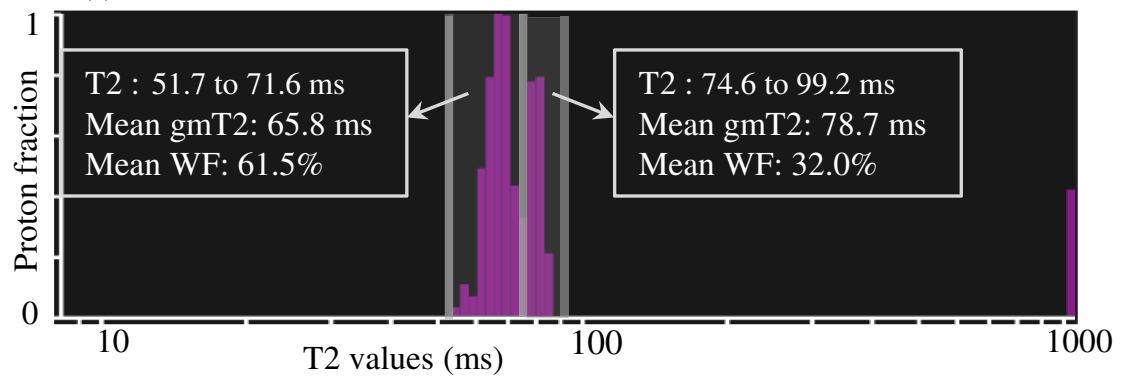

(c)

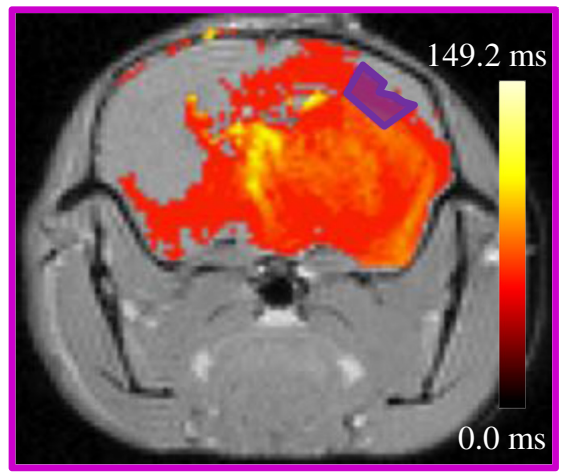

(e)

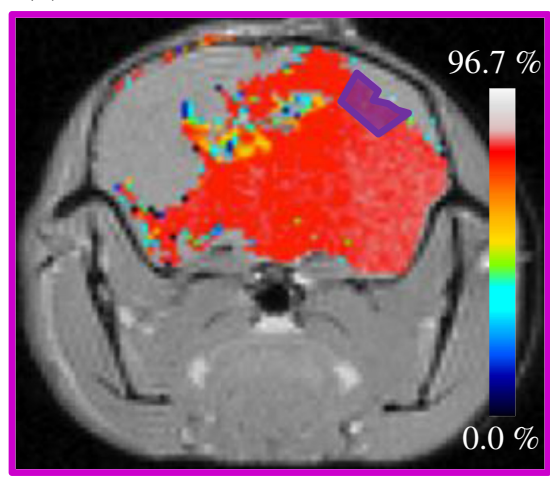
. 


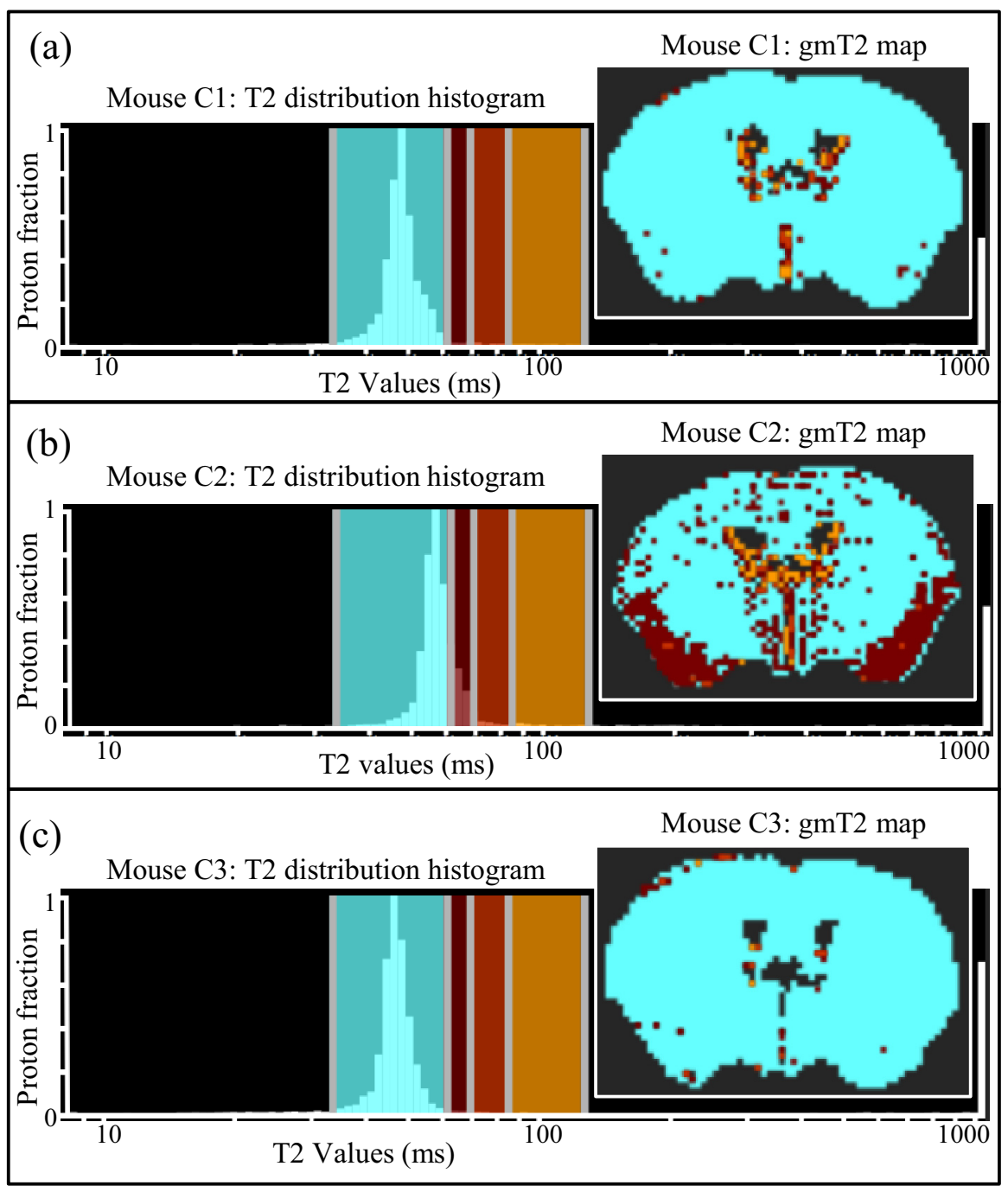

Fig. 6 (a) to (c) Segmented T2 distribution histograms and corresponding gmT2 maps for controls $\mathrm{C} 1$ to C3. Each T2 distribution histogram is divided into four T2 bands: 30.0 to $60.8 \mathrm{~ms}$ (T2 band 1, cyan); 63.4 to $71.6 \mathrm{~ms}$ (T2 band 2, maroon); 74.6 to $84.3 \mathrm{~ms}$ (T2 band 3, red); and 87.8 to $149.2 \mathrm{~ms}$ (T2 band 4, orange). The gmT2 maps are segmented into four regions, one for each T2 band.

range (30.4 to $60.8 \mathrm{~ms}$, outlined in blue). The other parts of the brain corresponded to 63.4 to $168.6 \mathrm{~ms}$, T2 values past the normal range of GM, as observed in Fig. 5(c). There, the gmT2 of the highlighted tissues were in the 0 to $149.2 \mathrm{~ms}$ range. These tissues were later found to contain tumor cells by histological analysis (see Sec. 3.5). Figures 5(d) and 5(f) display the WF maps generated for the same T2 ranges for MG1. Portions of the brain were highlighted for the $\mathrm{T} 2$ range of 30.4 to $60.8 \mathrm{~ms}$ with mostly homogeneous distribution [Fig. 5(d)]. Other regions of the brain corresponding to higher T2 values (outlined in purple) displayed a heterogeneous WF distribution in Fig. 5(e).

\subsection{Quantitative Characterization of Pathological Tissues}

Figure 5(f) displays the local T2 distribution histogram computed from the ROI shown on the gmT2 and WF maps of MG1 [Figs. 5(c) and 5(e)]. The histogram showed a heterogeneous T2 distribution with two distinctive peaks. The first peak (51.7 to $71.6 \mathrm{~ms}$ ) had a mean gmT2 of $65.8 \mathrm{~ms}$ with
WF of $61.5 \%$; and the second peak (74.6 to $99.2 \mathrm{~ms}$ ) had a mean gmT2 of $78.7 \mathrm{~ms}$ with WF of $32.0 \%$. The remaining $6.5 \%$ of the ROI signal corresponded to the CSF peak.

\subsection{T2-Based Segmentation of Normal and Pathological Tissues}

Figures 6 and 7 show results from T2-based segmentation of normal and pathological tissues in the control (C1 to $\mathrm{C} 3$ ) and glioma (MG1 to MG3) groups, respectively. For each mouse brain, the following $\mathrm{T} 2$ ranges were defined in the $\mathrm{T} 2$ distribution histograms: T2 band 1: 30.4 to $60.8 \mathrm{~ms}, \mathrm{~T} 2$ band $2: 63.4$ to $71.6 \mathrm{~ms}$, T2 band 3: 74.6 to $84.3 \mathrm{~ms}$, and T2 band 4: 87.8 to $149.2 \mathrm{~ms}$. The gmT2 map of each mouse brain was segmented into four regions, one for each $\mathrm{T} 2$ band. These $\mathrm{T} 2$ bands and the corresponding regions are colored in cyan, maroon, red, and orange, respectively. ROIs were drawn individually for each segmented region for further analysis. Tables 1 and 2 summarize the gmT2 and the WF data for the control and the glioma group, respectively. These results demonstrate an alternative analysis method of QuantitativeT2. 
(a)

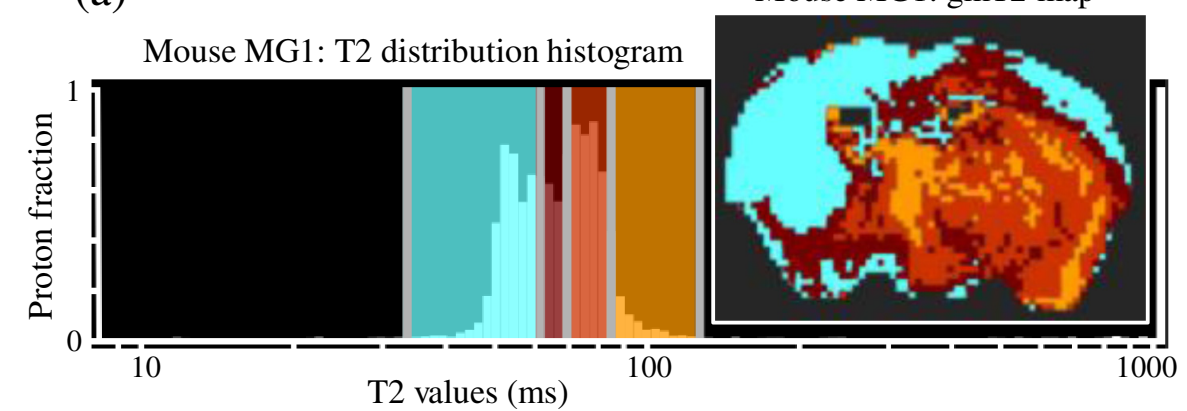

(b)

$\mathrm{T} 2$ values $(\mathrm{ms})$

Mouse MG2: gmT2 map

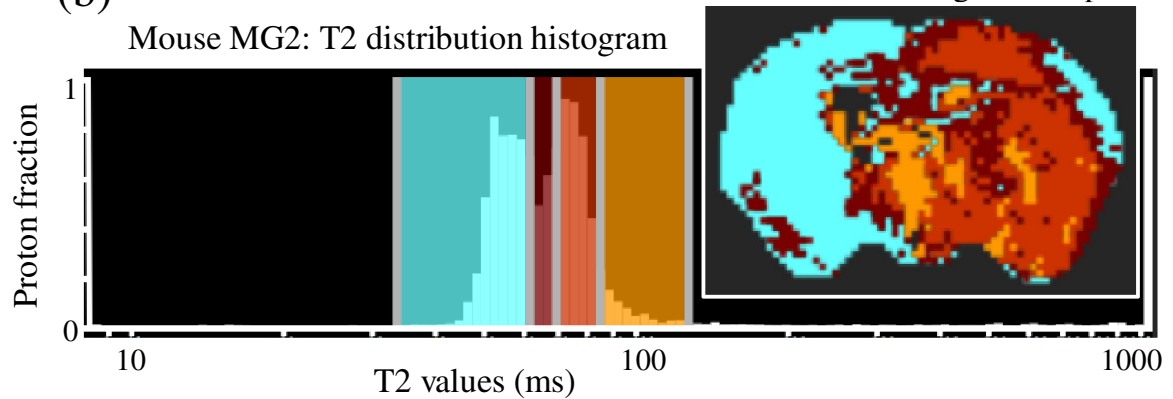

(c)

$\mathrm{T} 2$ values (ms)

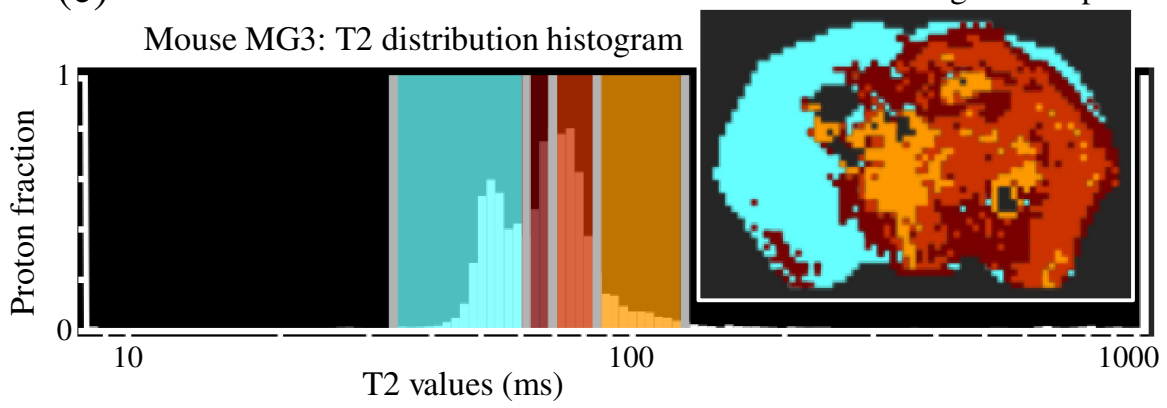

Fig. 7 (a) to (c) Segmented T2 distribution histograms and corresponding gmT2 maps for malignant glioma (MG) induced mouse brain scans, MG1 to MG3. Each T2 distribution histogram is divided into four T2 bands: 30.4 to $60.8 \mathrm{~ms}$ (T2 band 1, cyan); 63.4 to $71.6 \mathrm{~ms}$ (T2 band 2, maroon); 74.6 to $84.3 \mathrm{~ms}$ (T2 band 3, red); and 87.8 to $149.2 \mathrm{~ms}$ (T2 band 4, orange). The gmT2 maps are segmented into four regions, one for each T2 band.

\subsection{Comparison of qT2 with Histology}

For validation purpose, the results obtained by QuantitativeT2 were compared against histology.

The histology stains of the control group $\mathrm{C} 1$ to $\mathrm{C} 3$ revealed no tumor cells (data not shown). Figure 8(a) shows that the four color-coded regions in the gmT2 map of the glioma-bearing mouse brain, MG1, correspond with varying tumor cell densities, when visually compared with Fig. 8(b). Here density of brown stain represents tumor cell population. At 40x magnification [Figs. 8(c)-8(f)], the increase in tumor cell population with increased T2 times is striking. The histological analysis also confirmed that the BTICs were initially implanted at the central mass of the pathological tissue (highlighted in orange in the gmT2 map). In Figs. 8(g)-8(j), the nuclei are stained blue and the extracellular matrix stained pink. Increasing tumor cell density was observed with the increase in $\mathrm{T} 2$ for the regions corresponding to T2 bands 1, 2, and 3. Degeneration of the extracellular matrix was observed in the regions corresponding to the highest $\mathrm{T} 2$ time, T2 band 4 [Fig. 8(j)]. Similar results were observed in brain sections stained for nestin or H\&E from all three mice with glioma.
The histology sections stained for nestin were later quantified and analyzed using the ACIS III automated cellular imaging system for both control and tumor-bearing mice. For each whole brain section, histological regions corresponding to the $\mathrm{T} 2$ bands listed above were identified and the average

Table 1 The average geometric mean T2 (gmT2) and water fraction (WF) computed for two regions of interest (ROIs) corresponding to the first two T2 bands in the segmented T2 distribution histogram of controls $\mathrm{C} 1$ to $\mathrm{C} 3$ in Figs. 6(a)-6(c).

\begin{tabular}{lccccc}
\hline & \multicolumn{2}{c}{ Geometric mean T2 $(\mathrm{ms})$} & & \multicolumn{2}{c}{ Water fraction (\%) } \\
\cline { 2 - 3 } \cline { 5 - 6 } T2 band & 1 & 2 & & 1 & 2 \\
\hline ROI 1 & $49.2 \pm 4.1$ & $72.6 \pm 7.6$ & & $83.7 \pm 1.4$ & $1.5 \pm 0.8$ \\
ROI 2 & $49.9 \pm 3.9$ & $65.4 \pm 2.1$ & & $79.3 \pm 5.3$ & $5.8 \pm 3.9$ \\
\hline
\end{tabular}

Note: T2 band 1: 30.4 to $60.8 \mathrm{~ms}$, corresponds to ROI 1. T2 band 2: 63.4 to $71.6 \mathrm{~ms}$, corresponds to ROI 2 . 
Table 2 The average gmT2 and WF computed for four ROls corresponding to the four T2 bands in segmented T2 distribution histograms of the malignant glioma (MG) induced mouse brain scans MG1 to MG3 in Figs. 7(a)-7(c).

\begin{tabular}{|c|c|c|c|c|c|c|c|c|}
\hline \multirow[b]{2}{*}{ T2 band } & \multicolumn{4}{|c|}{ Geometric mean T2 (ms) } & \multicolumn{4}{|c|}{ Water fraction (\%) } \\
\hline & 1 & 2 & 3 & 4 & 1 & 2 & 3 & 4 \\
\hline ROI 1 & $52.1 \pm 0.7$ & $67.4 \pm 1.1$ & $74.6 \pm 5.1$ & $100.1 \pm 11.4$ & $68.8 \pm 1.7$ & $9.7 \pm 2.3$ & $6.2 \pm 1.3$ & $2.4 \pm 1.6$ \\
\hline $\mathrm{ROI} 2$ & $57.3 \pm 1.1$ & $67.5 \pm 0.6$ & $77.7 \pm 1.3$ & $92.2 \pm 3.4$ & $22.7 \pm 5.5$ & $47.9 \pm 9.8$ & $17.2 \pm 3.1$ & $2.5 \pm 1.6$ \\
\hline ROI 3 & $60.6 \pm 1.4$ & $69.2 \pm 0.3$ & $78.4 \pm 0.4$ & $92.2 \pm 3.7$ & $4.5 \pm 3.5$ & $28.9 \pm 5.1$ & $49.9 \pm 11.9$ & $7.4 \pm 4.6$ \\
\hline ROI 4 & $59.9 \pm 0.4$ & $69.1 \pm 1.0$ & $78.2 \pm 0.7$ & $103.9 \pm 5.0$ & $1.2 \pm 0.9$ & $20.2 \pm 9.5$ & $23.0 \pm 12.5$ & $39.2 \pm 10.1$ \\
\hline
\end{tabular}

Note: T2 band 1: 30.4 to $60.8 \mathrm{~ms}$; T2 band 2: 63.4 to $71.6 \mathrm{~ms}$; T2 band 3: 74.6 to $84.3 \mathrm{~ms}$; T2 band 4: 87.8 to $149.2 \mathrm{~ms}$. ROls 1 to 4 correspond to T2 bands 1 to 4 .

percentages of brown area (tumor cell cytoplasm) were calculated for these regions. Figure 9 demonstrates the nestin stain percentages in normal brains for the control groups $(\mathrm{C} 1$ to $\mathrm{C} 3)$ and BTIC tumor-bearing brains for the experimental group (MG1 to MG3). The T2 time increased, from 30.4 to $84.3 \mathrm{~ms}$ as the nestin stain\% increased, and therefore, the tumor cell density increased. However, nestin $\%$ decreased in brain regions with the longest T2 times (87.8 to $149.2 \mathrm{~ms}$ ). The data trends of nestin percentages were very strongly correlated $(p<0.001)$ according to Spearman's rank correlation coefficient $^{31}$ among the MG tumor-bearing brains, MG1, MG2, and MG3.

\section{Discussion and Conclusion}

Our voxel-based analysis method has enhanced the spatial resolution of qT2 analysis compared to ROI-based approaches. It now permits visual interpretation of the general $\mathrm{T} 2$ properties of all brain tissues in the scanned slice. Long T2 times are readily identified in the histogram, allowing easy localization of the corresponding anatomical regions. It has enabled the identification of T2 ranges of interest exhibiting subtle differences-these ranges would likely remain unidentified using the traditional ROI-based approaches. QuantitativeT2 has combined interactive computation and presentation of gmT2 and WF parametric maps. Two different properties-water microcompartment structure by gmT2 and partial voluming among these compartments by WF-are now revealed simultaneously by this arrangement. We have also improved the contrast and appearance of parametric maps by flexible color mapping and window leveling algorithms. The local ROI analysis tool has been effective for the isolation and characterization of specific tissues by gmT2 and WF measurements. Overall, we have improved the interpretation of qT2 results with a faster, easier, and intuitive workflow.

QuantitativeT2 is free and released as an open-source code. It can process the raw MR data from many manufacturers. It integrates mathematical processing, visual displays, and interactive analysis functionalities in one platform, such that no other software is required for data processing. It is also time-efficient; it completes qT2 analysis of $128 \times 128$ scan matrix in $<2 \mathrm{~s}$ on a desktop/laptop computer running Mac OS X 10.6 or newer. Yoo et al. recently described a method for qT2 analysis that leveraged multicore CPU and graphical processing units to reduce computation time. ${ }^{32}$ Their work is highly complementary, and different from ours, in several important ways. Their work is geared toward rapid, noninteractive, batch processing of multiple exams and is focused on production of static MWF maps. Our tool is designed for a dynamic workflow and interactive mapping of gmT2 and WF.

In the literature, qT2 MRI has been shown effective for analyzing multiple neurological conditions, such as tumor, ${ }^{15}$ multiple sclerosis, ${ }^{16}$ and phenylketonuria. ${ }^{19}$ In the current study, we have analyzed MG, one of the most common primary central nervous system tumors in adults. In conventional MRI, a long T2 is observed in regions of increased tissue water and blood volume, as well as in areas where brain tissue texture has been lost from gliosis. ${ }^{33}$ In a rat glioblastoma model, both short T2 $(20.7 \pm 5.4 \mathrm{~ms})$ and long T2 (76.4 $\pm 9.3 \mathrm{~ms})$ were observed in tumor ROIs, whereas a single T2 component $(48.8 \pm 2.3 \mathrm{~ms})$ was observed for normal GM. ${ }^{23}$ In the latter study, the ROI-based approaches used resulted in coarser spatial resolution and reduced detail in T2 histograms. In contrast, our voxel-based analysis method has shown that the T2 times between 30 and $150 \mathrm{~ms}$ are related to cellular density and the integrity of the extracellular matrix. The genotypic and phenotypic changes in MG are expected to result in high cellular density and matrix degeneration, ${ }^{34}$ which is in accordance with our results. qT2 mapping has been recommended for improved monitoring of glioblastoma patients under therapy. ${ }^{35}$ The analytical advancement from the conventional MRI to our analysis approach is clearly noticeable by comparing Fig. 3 with Fig. 5. The PD and T2 MRI in Fig. 3, which are applied in clinical settings, identify the pathological region but fail to isolate the tumor from normal tissues. Gray-scale intensity is the only information available for further diagnosis. For the same MR slice, voxel-based multiexponential MRI clearly delineates the tumor and provides quantitative information on tumor infiltration. The gmT2 and WF maps in Fig. 5 provide anatomical information specific to the progression of MG, which may assist in cancer staging. Likewise, QuantitativeT2 may also be useful for analyzing other neurological conditions and qT2 MRI data in general.

Due to technical constraints on data availability for human brain pathology, our method has been validated in a mouse model of MG up until the present date. The commonly used qT2 sequence scans one slice at a time, which is inadequate for patient screening. Currently available human qT2 data are acquired in research facilities and not clinical centers, which lack infrastructure to obtain the time-consuming multiecho sequences from all patients. Recently, promising progress has been made using new pulse sequences that accelerate multiecho 
(a)
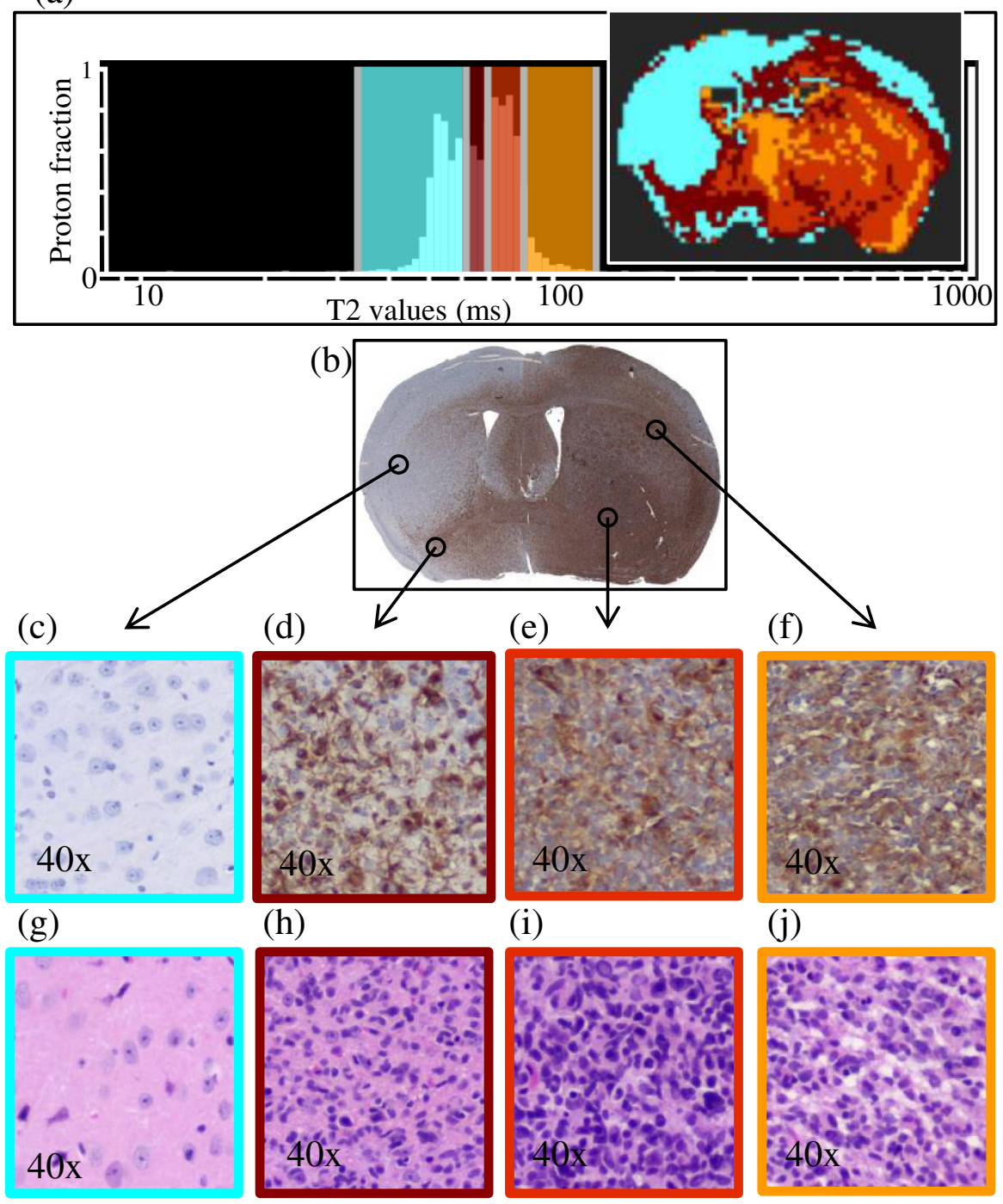

Fig. 8 Comparative analysis of qT2 results and histology for MG-induced brain slice. (a) The color-coded T2 distribution histogram and the corresponding segmented gmT2 map of MG1. The T2 bands are 30.4 to $60.8 \mathrm{~ms}$ (cyan); 63.4 to $71.6 \mathrm{~ms}$ (maroon); 74.6 to $84.3 \mathrm{~ms}$ (red); and 87.8 to $149.2 \mathrm{~ms}$ (orange). The T2 in the first band are normal values for gray matter, and the region colored in cyan indicates normal brain tissues. The other T2 bands hold increased T2 due to pathology and the corresponding regions indicate tumor tissues at different stages of development. (b) A histology section from the same magnetic resonance (MR) slice in mouse MG1 stained with an antibody for human nestin, where the density of brown stain indicates the tumor cell population. (c) to (f) Higher magnification (40x) images of the histological section corresponding to the four T2 bands: (c) shows normal tissue corresponding to cyan T2 band; (d) shows presence of tumor cells in tissue corresponding to maroon T2 band; (e) and (f) show increasing tumor cell population for red and orange T2 bands, respectively. (g) to (j) Higher magnification (40x) images of another histology section within MR scan slice stained with hematoxylin and eosin (H\&E) from mouse MG1 corresponding to the four T2 bands: (g) shows tissue with few normal cells for cyan T2 band, (h) and (i) show increasing cell population for maroon and red T2 bands, respectively, and (j) shows increased cell population and extracellular matrix degradation. The cell nuclei are colored blue and the extracellular matrix is colored pink.

data acquisition, while providing information from contiguous slices. ${ }^{36,37}$ Combining the scans of multiple slices, these new sequences also produce significantly large data. The new pulse sequences may encourage the clinical application of qT2 only if there is an efficient analysis tool-QuantitativeT2-to process the data and visually integrate the qT2 analysis results. In fact, QuantitativeT2 has been validated against existing qT2 analysis methods and normal human data. ${ }^{38}$
One limitation of MRI is its inherent low sensitivity level for identifying individual tumor cells. It can be argued that a very low concentration of tumor cells can remain unnoticed by voxelbased qT2 or T2-based segmentation as observed in the corpus callosum of the contralateral cerebral hemisphere in Fig. 8(b). This was due to the overwhelming effect of the normal tissue for a voxel volume $(0.15 \times 0.15 \times 0.75 \mathrm{~mm})$. However, the heterogeneous WF map of this region in Fig. 5(b) shows $\sim 70$ to $80 \%$ 


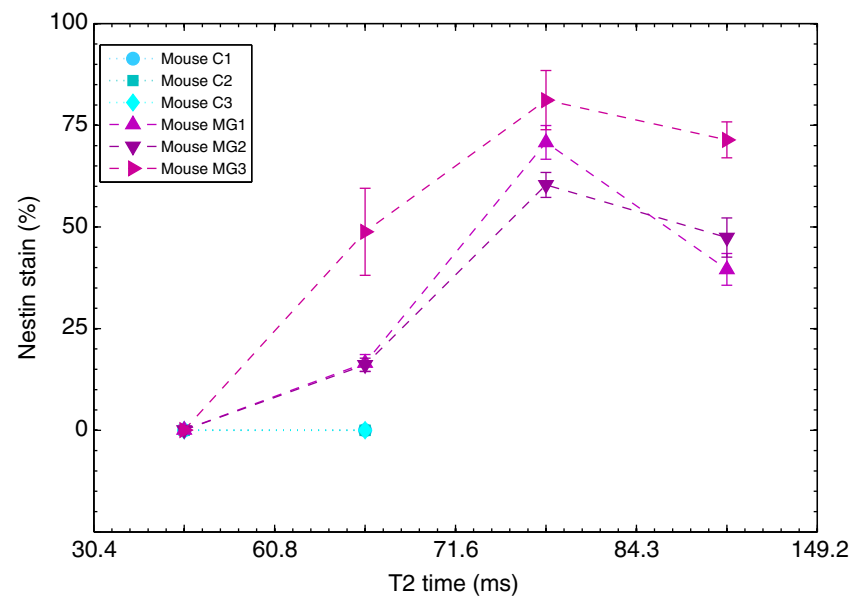

Fig. 9 The nestin stain (brown area) percentages for the ROls corresponding to four T2 bands: 34.0 to $60.8 \mathrm{~ms} ; 63.4$ to $71.6 \mathrm{~ms} ; 74.6$ to $84.3 \mathrm{~ms}$; and 87.8 to $149.2 \mathrm{~ms}$ computed using ACIS III automated cellular imaging system. $\mathrm{C} 1, \mathrm{C} 2$, and $\mathrm{C} 3$ present data from normal mouse brains. These results are identical; the data from $\mathrm{C} 1$ and $\mathrm{C} 2$ are overlapped by the data from C3. MG1, MG2, and MG3 present data from the mouse brains with MG tumors. The T2 time increases, from 30.4 to $84.3 \mathrm{~ms}$, as the nestin stain\% increases, and therefore, the tumor cell density increases. However, nestin\% decreases in brain regions with the longest T2 times ( 87.8 to $149.2 \mathrm{~ms}$ ). H\&E stains in these regions indicate they had degraded extracellular matrices compared to the other regions.

of WF for the prominent tissue and highlights the importance of further analysis. Perhaps the quantitative ROI characterization [shown in Fig. 5(f)] would provide more information on the tumor cells in that region. The strong correlation among the MG data nestin percentages proves it beyond doubt that the MG tumor infiltration follows a unique behavior in all three mice presented here as shown in Fig. 9. The tumor infiltration is related to $\mathrm{T} 2$ alterations, which are detectable by voxel-based qT2 analysis, when the tumor cells are detectable within MRI hardware capacity.

In conclusion, we developed QuantitativeT2-a software package for simple and real-time voxel-based qT2 analysis. QuantitativeT2 brings significant advancement to existing qT2 MRI technology. It provides a novel and interactive workflow for identification and characterization of $\mathrm{T} 2$ distribution histograms along with gmT2 and WF parameter maps. With it, we identified structural changes in glioma progression in relation to T2 variations. Being open-source, QuantitativeT2 should promote the use of qT2 analysis in research as well as in diagnostics.

\section{Acknowledgments}

This project was funded by Alberta Innovates Health Solutions, Alberta Innovates Technology Futures, Alberta Cancer Foundation, and Southern Alberta Cancer Research Institute. Thanks to Mark Simpson and Jeff Packer for helpful advice and support for software development.

\section{References}

1. K. P. Whittall et al., "In vivo measurement of $\mathrm{T} 2$ distributions and water contents in normal human brain," Magn. Reson. Med. 37(1), 34-43 (1997).

2. S. Meiboom and D. Gill, "Modified spin-echo method for measuring nuclear relaxation times," Rev. Sci. Instrum. 29, 688 (1958).
3. K. P. Whittall, A. L. MacKay, and D. K. B. Li, "Are mono-exponential fits to a few echoes sufficient to determine T2 relaxation for in vivo human brain?," Magn. Reson. Med. 41(6), 1255-1257 (1999).

4. A. MacKay et al., "Insights into brain microstructure from the T2 distribution," Magn. Reson. Imaging 24(4), 515-525 (2006).

5. L. Vidarsson et al., "Linear combination filtering for T2-selective imaging of the knee," Magn. Reson. Med. 55(5), 1191-1196 (2006).

6. R. S. Menon and P. S. Allen, "Application of continuous relaxation time distributions to the fitting of data from model systems and excised tissue," Magn. Reson. Med. 20(2), 214-227 (1991).

7. C. S. Poon and R. M. Henkelman, "Practical T2 quantitation for clinical applications," J. Magn. Reson. Imaging 2(5), 541-553 (1992).

8. M. Lupu, C. D. Thomas, and J. Mispelter, "Retrieving accurate relaxometric information from low signal-to-noise ratio 23Na MRI performed in vivo," C. R. Chim. 11(4-5), 515-523 (2008).

9. S. J. Graham, P. L. Stanchev, and M. J. Bronskill, "Criteria for analysis of multicomponent tissue T2 relaxation data," Magn. Reson. Med. 35(3), 370-378 (1996).

10. T. H. Storås et al., "Prostate magnetic resonance imaging: multiexponential T2 decay in prostate tissue," J. Magn. Reson. Imaging 28(5), 1166-1172 (2008).

11. G. Mulas et al., "A new magnetic resonance imaging approach for discriminating Sardinian sheep milk cheese made from heat-treated or raw milk," J. Dairy Sci. 96(12), 7393-7403 (2013).

12. M. H. Oztop et al., "Using multi-slice-multi-echo images with NMR relaxometry to assess water and fat distribution in coated chicken nuggets," LWT - Food Sci. Technol. 55(2), 690-694 (2014).

13. A. Mackay et al., "In vivo visualization of myelin water in brain by magnetic resonance," Magn. Reson. Med. 31(6), 673-677 (1994).

14. B. Mädler et al., "Is diffusion anisotropy an accurate monitor of myelination?: Correlation of multicomponent T2 relaxation and diffusion tensor anisotropy in human brain," Mag. Reson. Imaging 26(7), 874-888 (2008).

15. L. R. Schad et al., "Multiexponential proton spin-spin relaxation in MR imaging of human brain tumors," J. Comput. Assist. Tomogr. 13(4), 577-587 (1989).

16. C. Laule et al., "Myelin water imaging of multiple sclerosis at $7 \mathrm{~T}$ : correlations with histopathology," Neurolmage 40(4), 1575-1580 (2008).

17. E. E. Odrobina et al., "MR properties of excised neural tissue following experimentally induced demyelination," NMR Biomed. 18(5), 277-284 (2005).

18. C. Laule et al., "MR evidence of long $\mathrm{T} 2$ water in pathological white matter,' J. Magn. Reson. Imaging 26(4), 1117-1121 (2007).

19. S. M. Sirrs et al., "Normal-appearing white matter in patients with phenylketonuria: water content, myelin water fraction, and metabolite concentrations," Radiology 242(1), 236-243 (2007).

20. T. A. Bjarnason et al., "Quantitative T2 analysis: the effects of noise, regularization, and multivoxel approaches," Magn. Reson. Med. 63(1), 212-217 (2010).

21. A. L. M. Johnston et al., "The p75 neurotrophin receptor is a central regulator of glioma invasion," PLoS Biol. 5(8), e212 (2007).

22. B. Blasiak et al., "Detection of $\mathrm{T} 2$ changes in an early mouse brain tumor," Magn. Reson. Imaging 28(6), 784-789 (2010).

23. R. D. Dortch et al., "Evidence of multiexponential T2 in rat glioblastoma," NMR Biomed. 22(6), 609-618 (2009).

24. J. J. P. Kelly et al., "Proliferation of human glioblastoma stem cells occurs independently of exogenous mitogens," Stem Cells 27(8), 1722-1733 (2009).

25. L. Wang et al., "Gamma-secretase represents a therapeutic target for the treatment of invasive glioma mediated by the p75 neurotrophin receptor," PLoS Biol. 6(11), e289 (2008).

26. R. J. H. Lawson, Solving Least Square Problems, Prentice-Hall, Englewood Cliffs, NJ (1974).

27. K. P. Whittall and A. L. MacKay, "Quantitative interpretation of NMR relaxation data," J. Magn. Reson. 84(1), 134-152 (1989).

28. T. A. Bjarnason et al., "Temporal phase correction of multiple echo T2 magnetic resonance images," J. Magn. Reson. 231(0), 22-31 (2013).

29. K. P. Whittall et al., "Normal-appearing white matter in multiple sclerosis has heterogeneous, diffusely prolonged T2," Magn. Reson. Med. 47(2), 403-408 (2002).

30. A. K. Deguet, CISST Software Build Instruction, 2010, https://trac.lcsr .jhu.edu/cisst (3 May 2013). 
31. S. Siegel, Nonparametric Statistics for the Behavioral Sciences, p. 312, McGraw-Hill, New York, NY (1956).

32. Y. Yoo et al., "Fast computation of myelin maps from $\mathrm{MRI} \mathrm{T}_{2}$ relaxation data using multicore CPU and graphics card parallelization," J. Magn. Reson. Imaging 41(3), 700-707 (2014).

33. J. Oh et al., "Quantitative apparent diffusion coefficients and $\mathrm{T} 2$ relaxation times in characterizing contrast enhancing brain tumors and regions of peritumoral edema," J. Magn. Reson. Imaging 21(6), 701-708 (2005).

34. T. Demuth and M. Berens, "Molecular mechanisms of glioma cell migration and invasion," J. Neuro-Oncol. 70(2), 217-228 (2004).

35. E. Hattingen et al., "Quantitative T2 mapping of recurrent glioblastoma under bevacizumab improves monitoring for non-enhancing tumor progression and predicts overall survival," Neuro-Oncology 15(10), 1395-1404 (2013).

36. C. Laule et al., "In vivo multiecho $\mathrm{T} 2$ relaxation measurements using variable TR to decrease scan time," Magn. Reson. Imaging 25(6), 834839 (2007).

37. S. M. Meyers et al., "Reproducibility of myelin water fraction analysis: a comparison of region of interest and voxel-based analysis methods," Magn. Reson. Imaging 27(8), 1096-1103 (2009).

38. T. S. Ali et al., "A novel method to visualize quantitative T2 MRI data: qT2-View," Int. J. CARS 4(Suppl 1), S352-S353 (2009).

Tonima Sumya Ali is a PhD student of medical physics at the Queensland University of Technology. Her research focuses on micro MRI, MR image processing and quantitative MRI for cartilage structure and osteoarthritis assessment. She received her BSc degree in electrical engineering from the University of Alberta and her MSc degree in biomedical engineering from the University of Calgary.
Thorarin Albert Bjarnason is a CCPM certified diagnostic radiological medical physicist working for Interior Health, British Columbia, Canada. His current research interests include planar $x$-ray and CT quality control, radiation safety, patient dose optimization, and image quality assessment.

Donna L. Senger is a research associate professor in oncology at the Cumming School of Medicine, University of Calgary, and a principle scientist for Arch Biopartners Cancer Therapeutics. She has a longstanding interest in the area of brain development and tumorigenesis and runs a multidisciplinary translational research group that focuses on defining the molecular characteristics of highly invasive glioma and developing novel therapeutic agents for brain tumor patients.

Jeff F. Dunn is a specialist in applying MRI and near-infrared spectroscopy in biomedical applications. Much of his work is in imaging development, brain, regulation of oxygenation and the impact of hypoxia. $\mathrm{He}$ is a professor and director of the Experimental Imaging Centre at the University of Calgary, home of a 9.4T MRI and a range of animal- and human-oriented optical equipment. He has 140 publications.

Jeffrey $\mathrm{T}$. Joseph, $\mathrm{PhD}$, is a professor of pathology at the University of Calgary, director of Neuropathology at Calgary Laboratory Services, and Burns and Berlin professor in dementia research at the Hotchkiss Brain Institute. His research is in neuropathology with a focus on neurodegenerative diseases and brain banking.

Joseph Ross Mitchell is a professor of radiology in the Mayo Clinic College of Medicine and chair of the Division of Medical Imaging Informatics, Department of Radiology, Mayo Clinic Arizona. He is also the founding scientist and co-founder of Calgary Scientific Inc. His research is focused on extracting information from medical images to improve the diagnosis, treatment, and monitoring of disease. 